\title{
NonREM Disorders of Arousal and Related Parasomnias: an Updated Review
}

\author{
Muna Irfan ${ }^{1} \cdot$ Carlos H. Schenck ${ }^{2}$. Michael J Howell ${ }^{3}$ \\ Accepted: 14 January 2021 / Published online: 1 February 2021 \\ (C) This is a U.S. government work and not under copyright protection in the U.S.; foreign copyright protection may apply 2021
}

\begin{abstract}
Parasomnias are abnormal behaviors and/or experiences emanating from or associated with sleep typically manifesting as motor movements of varying semiology. We discuss mainly nonrapid eye movement sleep and related parasomnias in this article. Sleepwalking (SW), sleep terrors (ST), confusional arousals, and related disorders result from an incomplete dissociation of wakefulness from nonrapid eye movement (NREM) sleep. Conditions that provoke repeated cortical arousals, and/or promote sleep inertia, lead to NREM parasomnias by impairing normal arousal mechanisms. Changes in the cyclic alternating pattern, a biomarker of arousal instability in NREM sleep, are noted in sleepwalking disorders. Sleep-related eating disorder (SRED) is characterized by a disruption of the nocturnal fast with episodes of feeding after arousal from sleep. SRED is often associated with the use of sedative-hypnotic medications, in particular the widely prescribed benzodiazepine receptor agonists. Compelling evidence suggests that nocturnal eating may in some cases be another nonmotor manifestation of Restless Legs Syndrome (RLS). Initial management should focus upon decreasing the potential for sleep-related injury followed by treating comorbid sleep disorders and eliminating incriminating drugs. Sexsomnia is a subtype of disorders of arousal, where sexual behavior emerges from partial arousal from nonREM sleep. Overlap parasomnia disorders consist of abnormal sleep-related behavior both in nonREM and REM sleep. Status dissociatus is referred to as a breakdown of the sleep architecture where an admixture of various sleep state markers is seen without any specific demarcation. Benzodiazepine therapy can be effective in controlling SW, ST, and sexsomnia, but not SRED. Paroxetine has been reported to provide benefit in some cases of ST. Topiramate, pramipexole, and sertraline can be effective in SRED. Pharmacotherapy for other parasomnias continues to be less certain, necessitating further investigation. NREM parasomnias may resolve spontaneously but require a review of priming and predisposing factors.
\end{abstract}

Key Words Parasomnia $\cdot$ sleepwalking $\cdot$ somnambulism $\cdot$ sleep terror $\cdot$ disorders of arousal $\cdot$ confusional arousal $\cdot$ sexsomnia $\cdot$ status dissociatus $\cdot$ parasomnia overlap disorder $\cdot$ restless legs syndrome

Muna Irfan

irfan007@umn.edu

Carlos H. Schenck

schen010@umn.edu

Michael J Howell

howe1020@umn.edu

1 Department of Neurology, Minneapolis Veterans Affairs Medical Center/ Pulmonary allergy, Critical Care and Sleep, University of Minnesota, Minneapolis, MN, USA

2 Department of Psychiatry, Hennepin Health Care, University of Minnesota, Minneapolis, MN, USA

3 Department of Neurology, Sleep Disorders Center, University of Minnesota Medical Center, Minneapolis, MN, USA

\section{Classification of parasomnias}

Parasomnias are typically classified by the sleep state out of which they arise, nonrapid eye movement sleep (NREM) and rapid eye movement sleep (REM). We will focus mainly on NREM and related parasomnias in our discussion. (see Table 1) [1].

NREM parasomnias include disorders of arousal: confusional arousals, sleepwalking, and sleep terrors. These behaviors emerge when the cortex incompletely arouses from deep NREM sleep often due to comorbid conditions that provoke repeated arousal and/or promote sleep inertia. Changes in the cyclic alternating pattern, a biomarker of arousal instability in NREM sleep, are noted in sleepwalking and related disorders [2].

Sleep-related eating disorder, currently classified in the International Classification of Sleep Disorders 3nd revision 
Table 1 Classification of parasomnias (excluding REM parasomnias) (1)

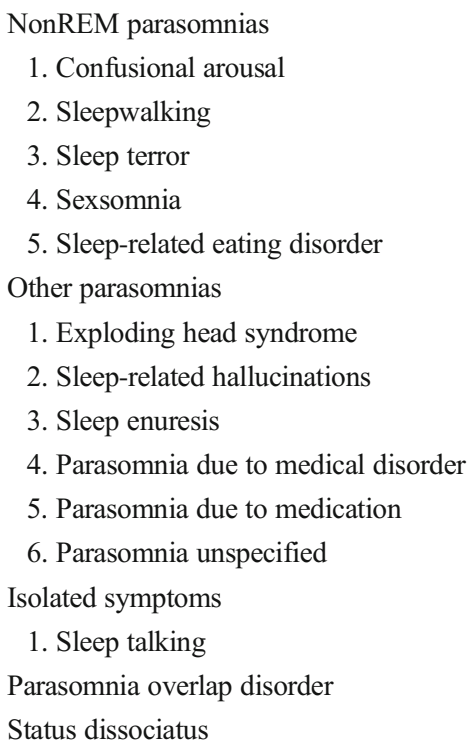

(ICSD-3) under nonREM parasomnia [1], is considered a subtype of SW.

Parasomnia overlap disorder, a combination of disorders of arousal and RBD, and status dissociatus, which are less commonly encountered among the parasomnia patient population, are currently classified as variants of RBD [1].

\section{NREM Parasomnias}

\section{Disorders of Arousal}

NREM sleep parasomnias are characterized by abnormal nocturnal behavior, impaired consciousness, and autonomic nervous system activation due to impaired arousal. This group of parasomnias, which is comprised of confusional arousal, sleepwalking, and sleep terror, is characterized by similar familial patterns and pathophysiological mechanisms. They typically arise from slow-wave (N3) NREM sleep [1]. Distinguishing features include duration, complexity, and type of behavior as well as degree of amnesia. Precipitating factors include conditions that result in either sleep fragmentation (noise), increased homeostatic sleep pressure (sedatives, sleep deprivations), or both (obstructive sleep apnea) (see Table 2) [1].

General criteria of disorders of arousal are listed in Table 3.

1. Confusional arousals $(\mathrm{CoA})$ are characterized by disoriented behavior during arousal from NREM sleep, often with vocalizations and poor recall of events the following day. While typically lasting less than $5 \mathrm{~min}$, episodes can occasionally last up to an hour. These prolonged episodes most commonly occur in the setting of polyneuropharmacy, in particular sedative-hypnotics. The behavior is typically benign however occasionally the patient can become aggressive and violent [1]. Diagnostic criteria are listed in Table 4.

2. Sleepwalking (SW) is the combination of ambulation with the persistence of impaired consciousness following arousal from sleep. Patients typically have amnesia and the behaviors are inappropriate, such as placing car keys in the refrigerator or rearranging furniture to nonfunctional locations. Attempting to arouse the patient is often difficult and may paradoxically worsen confusion and disorientation [1]. Table 5 enumerates the diagnostic criteria.

$\mathrm{SW}$ can become prolonged and/or dangerous. Alarming reports have described leaving the house, automobile driving, and sometimes discharge of loaded firearms. Similar to CoA, prolonged SW behaviors have been associated with sedative-hypnotic medications, in particular the benzodiazepine receptor agonists; however, unlike CoA, SW frequently occurs in the setting of Restless Legs Syndrome (see pathophysiology below).

3. Sleep terrors (ST) are episodes of intense fear initiated by a sudden cry or loud scream accompanied by increased autonomic nervous system activity. Most commonly, ST occur in preadolescent children. Parents often describe the patient as being inconsolable during events. In adults, ST can involve impulsively bolting out of bed without proper judgment in response to an imminent threatening image or dream fragment [1]. In such a clinical scenario, they likely also constitute SW. Severe injury or even death may result from leaping out of bed or jumping through a window. ST can last for greater than $5 \mathrm{~min}$ and attempts to abort an episode frequently result in even greater agitation. The criteria for ST are listed in Table 6.

4. Sexsomnia, considered a subtype of confusional arousal, is characterized by recurrent amnestic sexual behavior
Table 2 Factors provoking NREM parasomnia

\begin{tabular}{lll}
\hline Increased sleep fragmentation & Increased sleep inertia & Both \\
\hline Noise & Sleep deprivation & OSA \\
Pain & Circadian misalignment & Orexin dysfunction \\
RLS/PLM & Sedative-hypnotic medication & \\
\hline
\end{tabular}


Table 3 Criteria for DOA

1. Recurrent episodes of partial awakening

2. Inappropriate/absent responsiveness to redirecting efforts

3. Limited/lack of cognition or dream imagery

4. Partial/complete amnesia

5. Not caused by another sleep, mental, or medical condition/drug

ranging from masturbation to sexual intercourse [3, 4]. This category was formally described in 2007 with further updates and exploration in 2015 [5, 6]. The abnormal sexual activity occurs in slow-wave sleep or N3 akin to other NREM parasomnias. Studies have demonstrated male gender predilection. Evidence of treatment is limited in the literature, but clonazepam has shown benefit with some mixed results due to difficulty tolerating the drug [7]. Fluoxetine and trimipramine also provided relief in few cases [7]. The cases with coexistent OSA responded to treatment with CPAP or mandibular advancement device. The amnestic sexual behavior can result in relationship strife with potential forensic consequences (for extensive review, see [3-5].

5. Another variation is sleep-related eating disorder (see below), manifesting with dysfunctional nocturnal eating often leading to weight gain. Interestingly, nocturnal eating is a common nonmotor manifestation of RLS $[8,9]$ and the misdiagnosis and treatment of RLS as insomnia (with sedative-hypnotics) frequently leads to amnestic sleeprelated eating disorder [8]. Diagnostic criteria are listed in Table 7.

\section{Clinical Presentation}

Although NREM parasomnias peak in childhood, they are not uncommon in adults with a prevalence range between 1 and $4 \%[1,10]$. NREM parasomnia behaviors occur on a spectrum in terms of duration, autonomic activity, and impaired arousal. CoA are frequently of shorter duration compared to SW or ST. Prolonged (>60 min) episodes have been associated with sedative-hypnotic medications [11]. Regarding autonomic function, CoA and SW have less autonomic activation compared to ST, which are characterized by increased HR,

Table 4 Criteria for CoA

\begin{tabular}{ll}
\hline 1. & The criteria for DOA are met \\
2. & Episodes of confused behavior while patient is in bed \\
3. & Absence of terror or ambulation \\
\hline
\end{tabular}

Table 5 Criteria for SW

1. The criteria for DOA are met

2. Episodes associated with ambulation \& complex behavior out of bed

tachypnea, diaphoresis, and facial flushing. While all of the NREM parasomnias have impaired arousal, attempts to wake a patient from a ST often result in a paradoxical increase in agitation. NREM parasomnia patients are at least partially amnestic for nocturnal behaviors. Children with ST will not recall the dramatic events often to the bewilderment of concerned parents who witness the experiences [1]. Some of the clinical features are listed in Table 8.

Obstructive sleep apnea (OSA) and RLS are the most commonly identified precipitating factors in patients with SW [11, 12]. Other conditions that are associated with NREM parasomnias are also characterized by sleep fragmentation and/or increased homeostatic sleep pressure including the following: shift work, sedatives, environmental sleep disruption, and Periodic Limb Movements (PLMs) [13, 14].

Parasomnias are notoriously common in patients on sedative-hypnotic medications. One group of investigators noted a high frequency of SW and other amnestic complex behaviors among psychiatric patients who took benzodiazepine receptor agonist (BRA) medication [15]. These findings are consistent with other reports of abnormal nocturnal behavior induced by BRAs, in particular zolpidem $[11,16,17]$. In the setting of BRA induced parasomnias, the behaviors are often prolonged and can include amnestic nocturnal eating (SRED), sexual activity (sexsomnia), and sleep driving. These complex amnestic behaviors frequently occurred in the setting of CNS polypharmacy or supratherapeutic doses. Not unexpectedly there has been an increase in sleepassociated amnestic complex behavior in parallel to the contemporary rise in use of sedative-hypnotic medication [11] which ultimately led to inclusion of a black box warning by FDA in 2019 for careful monitoring and discontinuation of these agents in patients who experience a parasomnia after taking them.

Intriguingly many cases of BRA induced SW are noted to have comorbid RLS which could be easily misdiagnosed and treated as insomnia [8, 11]. Then, not surprisingly, BRAs, whose mechanism of action, with suppression of memory along with executive function, unleashes prolonged amnestic ambulating events by disinhibiting hippocampal and frontal lobe function $[8,11]$.

SW has also been associated with a variety of other medications and medical conditions. Implicated agents have included: central nervous system depressant sodium oxybate [18], antidepressants amitriptyline [19], buproprion [20], paroxetine [21], and mirtazapine [22], the mood stabilizer lithium [23], antipsychotics quetiapine [24], and olanzapine [25, 26], 
Table 6 Diagnostic criteria for ST

\begin{tabular}{ll}
\hline 1. & The criteria for DOA are met \\
2. & Episodes characterized by abrupt terror/alarming scream \\
3. & Intense fear and autonomic arousal (mydriasis, tachycardia, tachypnea, diaphoresis)
\end{tabular}

the antihypertensive agent metoprolol [27], the antiseizure agent topiramate [28], and the antibiotic fluoroquinolone [29]. Medical conditions associated with NREM parasomnias include: migraine [30], febrile illness [31], vitiligo [32], hyperthyroidism [33], as well as encephalitis and Stroke [1]. These diverse conditions and medications likely induce NREM parasomnias through a final common pathway. The exact mechanism of that pathway has not yet been determined. One interesting possible explanation, i.e. the serotonin hypothesis, will be described later.

\section{Pathophysiology}

SW and related disorders occur when there is an incomplete dissociation of NREM sleep into wakefulness. Two pathological processes may lead to this sleep-wake boundary dysfunction (see Table 2). First, phenomena that deepen sleep and enhance sleep inertia promote NREM parasomnias by impairing otherwise normal arousal mechanisms. Second, conditions that cause repeated cortical arousals lead to NREM parasomnias through sleep fragmentation. These abnormal arousals are often associated with the normal alternating arousal microstructure of NREM sleep, the cyclic alternating pattern (CAP) [2]. The complex amnestic behaviors that characterize these conditions are related to central pattern generators [34]. The isolated activation of these functional groups of motor neurons with a relative paucity of activity in brain regions that control executive function and memory account for the poor judgment and amnesia that characterize NREM parasomnias.

In the normal transition from light NREM sleep to wakefulness, consciousness emerges quickly, typically within seconds. The duration of normal arousal depends upon an intricate combination of variables including the following: duration of prior wakefulness, current sleep duration, depth of
NREM sleep, circadian rhythm phase, effects of sedating or stimulating medications, as well as multiple genetic and environmental factors. Stimuli of endogenous and exogenous origins activate neurons in the brainstem and the basal forebrain. These regions subsequently promote wakefulness through both direct activation of the cerebral cortex and by inhibiting thalamic reticular neurons, thus blocking spindle oscillations. These alerting phenomena lead to suppression of slow wave activity (SWA) and more predominant fast cortical activity appears compatible with wakefulness [35].

The speed of the conversion from NREM sleep to wakefulness depends upon the intensity of SWA. Most arousals into wakefulness arise from lighter stages (N1 or N2) of sleep with minimal SWA. The threshold for which stimulation is required to produce an awakening during light sleep is low. By comparison, the threshold for an awakening from deep NREM sleep (N3), characterized by nearly continuous SWA, is high and awakenings are typically prolonged [36]. Subsequently, sleep inertia during N3 sleep arousals is strong (subjectively referred to as "sleep drunkenness") and promotes a return to somnolence.

In NREM parasomnias, impaired arousal mechanisms and the persistence of sleep drive result in a failure of the brain to fully transition into wakefulness. Indeed, most SW and related disorders arise out of N3 sleep. Thus, sleep-promoting conditions such as sleep deprivation and sedative-hypnotic medication will lead to NREM parasomnias.

Conversely, disorders that lead to fragmented NREM sleep precipitate SW and other disorders of arousal by increasing arousal frequency. OSA, noise, and orexin dysfunction (cause of sleep instability in narcolepsy) all promote parasomnias by fragmenting NREM sleep. In fact, CoA can be precipitated in the sleep laboratory through sleep deprivation (which promotes SWA) combined with a sudden loud noise (see management below) [14].
Table 7 Diagnostic criteria for SRED

\begin{tabular}{ll}
\hline 1. & Recurrent episodes of nocturnal eating occurring after arousal from sleep \\
2. & One of the following \\
a & Consumption of peculiar forms/combination of food or inedible substances \\
b & Sleep-related potentially injurious behaviors performed in pursuit of food \\
c & Adverse health consequences from recurrent nocturnal eating \\
3. & Partial or complete loss of consciousness \\
4. & Not explained by other sleep, mental or medical disorder, substance use \\
\hline
\end{tabular}




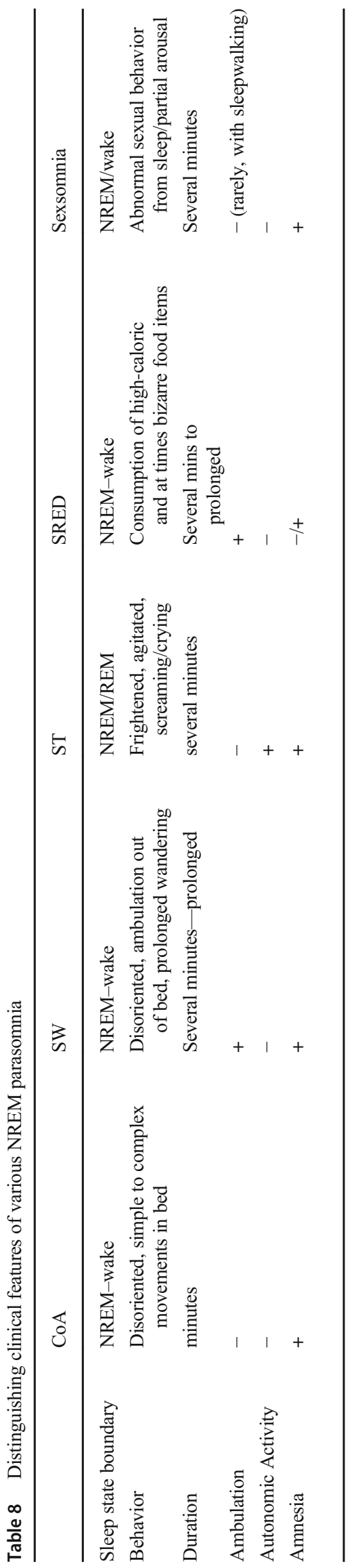

The chronic intermittent airway collapse in patients with OSA leads to NREM parasomnias through parallel mechanisms, sleep fragmentation as well as increased homeostatic sleep drive [2, 12, 33, 37].

Combinations of predisposing and precipitating factors frequently lead to SW. For example, CNS polypharmacy is often the setting for dangerous behavior such as sleep driving [33, 38]. Other examples include patients with OSA, who are prescribed sedative-hypnotic medication to assist with continuous positive airway pressure (CPAP) compliance. A similar situation often in patients with RLS misdiagnosed as having insomnia and subsequently treated with a BRA. As patients with RLS have a strong subconscious drive to ambulate, it is not unexpected that agents which suppress memory and executive function would lead to amnestic sleepwalking behaviors $[8,33]$.

A genetic component has also been suggested, supported by studies demonstrating high prevalence. of human leukocyte antigen (HLA) DQB1*05:01 and HLA DQB1*04 alleles in various nonREM parasomnias [13].

\section{Sleep Microarchitecture in NREM Parasomnias and the Cyclic Alternating Pattern}

In patients with nonREM parasomnia, evaluation of sleep demonstrated either normal distribution of NREM sleep and its individual sleep stages $[39,40]$ or slightly decreased N2 and higher N3 (slow wave) sleep [41] .

However, the microstructure of sleep in patients with NREM parasomnias often demonstrates several interesting findings. Commonly there is an increase in arousals, either related to PLMs, respiratory events or spontaneously [41, 42]. Autonomic activation, as measured by heart rate variability, precedes cortical and behavioral arousals [43]. Further, the density of slow-wave activity (SWA) during the early sleep cycles is relatively decreased compared to controls $[2,44]$. However, immediately preceding a confusional arousal, there is frequently an increase in SWA, commonly referred to as hypersynchronous delta (HSD) [41, 45]. The cyclic alternating pattern (CAP), a marker of NREM instability provides insight into these phenomena [46] (see below). Immediately after arousal, there is typically a persistent slowing of brain activity as measured by surface EEG [40, 47].

The CAP is an intrinsic oscillation throughout NREM sleep between periods of cortical arousal and quiescence throughout NREM sleep. This oscillation typically occurs every 20 to $40 \mathrm{~s}$ and provides the scaffolding for normal (such as $\mathrm{K}$ complexes and delta bursts) as well as pathological NREM phenomena (CA and SW events) [46]. SW/ST patients have an increased number of CAP cycles and a higher CAP rate, which is a measure of NREM instability [2, 39, 48]. Further, a subtype of cortical arousal in the CAP (phase A1) is characterized by hypersynchronous delta activity (HSD) and the majority of 
reports indicate SW, ST, and CoA are often preceded by a phase A1 run of HSD, indicating that these events are linked to the CAP $[41,45]$. Finally, it has been proposed that the CAP may be used as a biomarker for treatment response. In particular, resolution of CAP abnormalities in patients who are being treated for sleep disordered breathing is associated with a resolution of SW behaviors [46].

The increase in CAP activity noted in parasomnias (increased type A1 arousals and increased CAP rate) indicates underlying NREM instability. Thus, abnormal CAP activity is likely not the cause of parasomnias but rather a marker of a sleep destabilizing process. Candidate processes include neuropsychiatric disease, medications, subtle respiratory events, as well as innate genetic factors. In fact PLM's are also plausibly not directly causal to sleep instability but instead are a manifestation of unstable CAP [34]. These insights suggest that treatment of NREM parasomnias should be directed at resolving underlying sleep destabilizing processes and that the CAP changes (decrease in CAP rate and number of A1 events) may be used as a marker of treatment response.

Intracranial monitoring and neuroimaging in NREM parasomnia patients have indicated that the slowing of cortical activity after arousal is not diffusely distributed and that certain regions may become more activated/wake-like and coexist with regions that are in slow/NREM sleep-like activity. One report captured confusional arousal in a 20-year-old male who was undergoing intracerebral EEG monitoring for refractory epilepsy. The cingulate and motor cortices demonstrated an arousal followed by brain waves consistent with wakefulness while in parallel the fronto-parietal associative cortices had increased delta activity consistent with deep NREM sleep [49]. These findings were very similar to a SW event that was captured in a 16 yo male with cranial single photon emission computed tomography. In this case, the parasomnia was characterized by activation (increased regional cerebral blood flow) of motor coordination pathways with a relative paucity of activation in the frontal lobe [50].

\section{Central Pattern Generators and their Role in Parasomnias}

The behaviors that characterize parasomnias span a broad range from inaudible vocalizations to complex behaviors. It has been noted that these automatisms often resemble stereotyped behavior noted in other primates as well as more genetically distant mammalian and reptilian species [51]. In fact nocturnal subconscious behavior frequently resembles "primitive" activity such as: defensive postures, violent gestures, and sexual movement $[34,52]$. The presence of similar behaviors among animal groups with distant common ancestors suggests that they arise from shared brain structures, in particular subcortical regions, including the brainstem and spinal cord.
A unifying concept, the central pattern generators (CPG's), explains this spectrum of activity. CPGs are functional groups of neurons that give rise to subconscious patterns of motor activity.

CPGs require a certain amount of activation or disinhibition to generate stereotyped or quasi-stereotyped motor activity. This has been elegantly described as the facilitation of a "kinetic melody" [34]. During NREM parasomnias, intrinsic activation of an encoded motor plan occurs during the cortical arousal phase of the CAP (see above) [34]. Thus, any process, such as sleep apnea, that leads to NREM instability promotes more frequent CPG behavioral activation. External stimuli, such as a sudden noise can also promote activation of CPGs and in fact this technique is used to clinically induce SW and CoA (see management below).

Furthermore, during NREM parasomnias CPGs are activated in isolation from other brain structures that are important for normal wakeful behavior. In particular, imaging studies have revealed a paucity of activation in the dorsal lateral frontal cortex and hippocampal structures during NREM sleep [53]. Thus, with sudden arousals, the motor activity of SW (an expression of CPG activation) occurs in parallel with poor executive function and amnesia.

Importantly, by further impairing frontal lobe function, CPG behaviors are amplified in the setting of sedativehypnotic medication. This occurs through enhancement of GABAa activity and is related to dose and binding affinity [33]. By hindering cortical arousal, sedative-hypnotics impede the conscious, executive, "brake" on CPG behavior. Thus, elaborate inappropriate behaviors emerge as brain regions that encode motor behaviors are activated in parallel to frontal lobe inhibition. This is particularly relevant in the setting of pathological predisposed behaviors, such as the urge to ambulate in patients with RLS.

\section{Serotonin Theory of Parasomnias}

While the molecular pathophysiology of NREM parasomnias is poorly understood there is evidence that serotonergic pathways may be implicated [54]. First, serotonergic agents have been known to induce SW [23, 31] but conversely, these agents can also effectively treat other parasomnias, most notably ST [55]. Second, serotonin provides activation to motor neurons [56, 57]. Third, this motor activity may be dissociated from consciousness [54]. Fourth, serotonin activity is a plausible link between sleep disordered breathing and parasomnias as serotonin neurons are activated by hypercapnic acidosis [54, 57]. Fifth, disorders associated with SW such as migraine [30] and fever [31], are characterized by surges of serotonin [54]. It has thus been suggested that serotonergic neurons, activated by nocturnal respiratory events, may pathologically trigger CPG's resulting in sleep-related motor activity [54]. This is an intriguing hypothesis; however, further 
research is needed and any explanation should account for the discrepancy in the treatment between parasomnias.

\section{Alternative Theory of Sleep Terrors}

ST, while superficially similar to CoA and SW (in regard to abrupt arousal from SWS), may nevertheless originate in part from a distinctive neurophysiologic mechanism. In particular, it has been suggested that instead of an overlap between NREM and wakefulness as implicated in other NREM parasomnias, ST may represent a disorder of transition between deep NREM sleep (N3) and REM sleep. This theory would explain several unique features of ST: the high autonomic activity and the apparent vivid mentation (both from REM) combined with difficult arousability (from N3). Further, REM-NREM overlap may also explain the striking preponderance of ST in childhood as children have a greater proportion of both N3 and REM sleep. Intriguingly, the antidepressant medication paroxetine, an agent that can both induce SW [21] and treat ST [55], is a potent REM suppressor. Thus, if ST were due to pathological REM/NREM overlap, it would be expected that paroxetine would block these phenomena. Conversely, paroxetine would not be expected to have a therapeutic effect on SW, a wake/NREM phenomena. However, based on paroxetine's serotonergic effects it would be expected to potentially worsen SW [54] (see serotonin theory of parasomnias above).

\section{Sleep-Related Eating Disorder}

Under normal human physiological conditions, nighttime is characterized by a prolonged period of fasting associated with sleep. Energy homeostasis is maintained through the sleep period by alterations in metabolism and appetite modulation. This stands in contrast to fasting during sedentary wakefulness which demonstrates progressive hypoglycemia over $12 \mathrm{~h}$. The sleep-related fast is disrupted in SRED, characterized by recurrent episodes of nocturnal eating after arousal with adverse consequences. The episodes are described as occurring in an involuntary, compulsive, or "out of control manner". Often, patients describe an inability to return to sleep without eating and in this regard resemble other nocturnal compulsions that interfere with sleep such as restless legs syndrome (RLS). Amnestic SRED, like SW, is often related to sedative-hypnotic medication use, most commonly zolpidem. These cases are often characterized by prolonged episodes with elaborate and sometimes dangerous food preparation $[16,33,58,59]$.

\section{Clinical Presentation}

SRED is common (4-5\% prevalence) particularly among patients with other sleep disorders $[8,44,60]$. The most striking relationship is between SRED and RLS where both eating and motor symptoms frequently coexist and fluctuate in parallel. In a survey of 88 RLS patients who presented to a sleep disorder center, $61 \%$ had frequent nocturnal eating (NE) and $36 \%$ had SRED [8]. These findings are similar to a survey of 100 RLS patients who demonstrated a $33 \%$ prevalence of SRED [44]. Further, the mistreatment of RLS with benzodiazepine receptor agonists frequently induces amnestic SRED [40]. In contrast, dopaminergic therapy resolves NE and SRED in parallel with motor restlessness $[6,44]$ (see the relationship between SRED and RLS below).

At night SRED patients consume foods higher in carbohydrates and fats than typical daytime ingestion [61]. Weight gain is commonly reported $[1,62]$. The majority of patients describe chronic unrelenting daily symptoms that may persist for decades prior to pursuing treatment [62]. Nearly a quarter of SRED patients will experience greater than 5 episodes of nocturnal food ingestion [61]. The majority (60-83\%) of reported SRED cases are female and frequently coexists with daytime eating disorders [60-62].

Amnestic SRED, although common in many SRED cases, is especially common in the setting of sedative-hypnotic medications, in particular the benzodiazepine receptor agonists $[8$, $15,16,62]$. These unconscious episodes may include nonfood ingestions such as cigarettes, coffee grounds, or eggshells. Other patients will ingest substances they would otherwise avoid, such as patients with food allergies, diabetes, hyperlipidemia, or undergoing general anesthesia the next day [46, 63]. Patients may fall asleep with an oral bolus of food, which combined with the circadian decline in salivation places the patient at high risk of dental caries [46]. Finally, food preparation can include using the stove and/or oven in a haphazard manner increasing the risk for fires [46, 63].

SRED is usually associated with SW. Three series have reported comorbid SW in 48 to $65 \%$ of patients with SRED. SW without eating may precede SRED and then once nocturnal eating develops, it may become the predominant SW behavior $[15,62]$.

\section{Pathophysiology}

\section{SRED and Benzodiazepine Receptor Agonists}

Several early reports noted that amnestic nocturnal eating was associated with sedating psychotropic medications. A 1981 case report described amnestic nocturnal eating after initiating a combination of chlorpromazine, amitriptyline, and methyprylon [64]. Subsequently, SRED has been reportedly induced with triazolam, lithium, olanzapine, risperidone, zopiclone, and zaleplon $[25,46,65,66]$ as well as zolpidem extended-release formulation [67]. 
The majority of drug cases are related to zolpidem, a benzodiazepine receptor agonist. The first series of zolpidem associated SRED described five middle-aged patients, two of whom already had intermittent episodes of conscious nocturnal eating prior to starting zolpidem. Interestingly all 5 had a history of RLS. Soon after initiating zolpidem, each patient described amnestic nocturnal eating that stopped with discontinuation [16].

Further reports have strengthened the relationship between zolpidem and SRED. In a series of 1235 patients at an outpatient psychiatry clinic, the combination of zolpidem and antidepressants posed the greatest risk for SRED [15]. In another report of 29 sleepwalkers with frequent BRA use, approximately $2 / 3$ of patients described sleep-related eating behavior [68]. The vast majority of reports note improvement if not outright resolution once the agents were discontinued [16, 68].

SRED frequently occurs when, in a desperate attempt to initiate sleep, patients take supratherapeutic doses of zolpidem $[58,69]$.

BRAs enhance GABA activity at central GABA A receptors resulting in hypnotic phenomena [33]. As these agents suppress executive function it may be that zolpidem by itself, does not activate SRED but instead disinhibits the behavior in a patient population at risk for nocturnal feeding. Patients with RLS who are not on sedatives demonstrate a greater tendency towards wakeful, nonpathological nocturnal eating [8, 44]. Conversely, when on sedatives, particularly BRAs, patients with RLS frequently demonstrated amnestic SRED [8, 16, 67, 70]. (see section on RLS and SRED).

\section{Medication-Induced Amnesia}

Impaired consciousness, as a defining criterion for SRED, has evolved since complete or at least partial unawareness was necessary for diagnosis. In the original series of 32 SRED patients, $84 \%$ claimed an impaired recall [71]. Conversely, a subsequent report noted full awareness in all 26 patients after episodes of nocturnal eating in a sleep laboratory [61].

\section{The Relationship Between SRED and RLS}

In many cases of SRED, nocturnal food ingestion is best characterized as restless eating, i.e., in order to facilitate sleep. In this regard, nocturnal feeding behaviors bear a striking similarity to the motor symptoms of RLS. In fact, reports have described that nocturnal eating is pervasive among RLS patients including the original description by Ekbom in 1960 [8, 69, 70]. "They often have to get up and walk, "like a caged bear," to quote one of my patients, or they go into the kitchen and get something to eat" [72]. Further similarities in epidemiology, polysomnographic phenomena, clinical course, and treatment response are reviewed here and suggest an intimate relationship between nocturnal food ingestion and RLS.
RLS is a disorder affecting approximately 8 to $10 \%$ of the population and thus a common cause of sleep initiation and maintenance failure $[1,73,74]$. Further, while RLS is distinct from, it is commonly confused with psychophysiological insomnia (PI). Thus, it may be expected that many patients with RLS will be mistakenly treated with therapies designed to treat PI, agents such as BRAs.

Like SRED, RLS has a higher prevalence in women [1, 73]. Further, medication-induced SRED is more common in women $[58,69]$.

Similar to RLS [75], several features of SRED suggest an underlying dopamine dysfunction. First, dopamine mediates impulsive behaviors such as motor restlessness, smoking, and binge eating [75]. Second, a polysomnography (PSG) study of 35 SRED patients demonstrated that $77 \%$ had PSG confirmation of wakeful RLS and periodic limb movement during sleep [61]. Third, rhythmic masticatory muscle activity (RMMA) and bruxism, dopaminergic phenomena [56, 76] associated with RLS, are commonly seen in SRED [46, 61]. In the original SRED case series, prominent RMMA was described during NREM sleep and after arousals [46]. Recently, RMMA was found in 29 of 35 patients diagnosed with SRED during their PSG evaluations [61].

Recently, two investigations demonstrated a high prevalence of both SRED and nondysfunctional nocturnal eating in patients with RLS. A community survey of 100 RLS patients reveals a high prevalence of SRED in RLS (33\%) compared to normal population controls (1\%) [70]. The authors pondered whether the compulsive nocturnal eating was related to underlying RLS brain pathology or whether nocturnal eating was merely "killing time" as previously suggested. This question was addressed in another study of 130 patients with either RLS or PI who presented to a sleep disorders center. This report noted that $61 \%$ of RLS patients described either nondysfunctional NE (25\%) or SRED (36\%). Conversely, only $12 \%$ of patients with PI described NE and no patients met criteria for SRED. This study suggests that nocturnal eating in RLS is not merely "killing time" as PI patients were more likely to have prolonged ( $>5 \mathrm{~min}$ ) nightly awakenings (93\%) compared to patients with RLS (64\%) [8].

Intriguingly, the nocturnal feeding behavior of SRED closely resembles the motor activity of RLS. RLS is characterized by an underlying feeling (often poorly described) of discomfort in the lower extremities that compels the patient to move. Movement relieves the discomfort and sleep is unable to be reinitiated until this urge is addressed [77]. In SRED, patients state that after awakening from sleep, they have a compulsion to eat (often without hunger) that interferes with sleep maintenance. Subsequently, once food is ingested, the feeling abates and sleep may be reinitiated $[1,46,70]$.

Compulsive nocturnal eating is not unexpected as RLS patients often describe other nonmotor nocturnal urges [78]. Recently, 6 cases of nocturnal eating and nocturnal smoking 
were reported. 5 of the 6 cases either presented with or were noted to have RLS. Patients claimed that they would wake up and be unable to return to sleep without eating and/or smoking. In a follow-up study that investigated the prevalence of sleep-related smoking, RLS patients demonstrated an increased prevalence (12\%) compared to matched controls (2\%). Interestingly, among RLS patients with nocturnal smoking, SRED was common $(83 \%)$ and both phenomena often began simultaneously [78].

It has been debated whether SRED in RLS may be caused by dopaminergic drugs as these agents are known to trigger daytime impulsive behaviors such as gambling [79-81]. However, the preponderance of evidence suggests that dopamine agents are not the cause of SRED. First, dopamine agents suppress feeding behavior in animal models [82]. Second, a review of the original SRED series noted that dopaminergic therapy resolved the dysfunctional eating in 7 of 8 patients in whom the treatment was attempted [46]. Later, two cases of SRED were noted to resolve with levodopa (in combination with buproprion and trazodone) [83]. Third, in a separate survey of patients with both SRED and RLS, 10 patients reported that nocturnal eating emerged prior to or concomitant with motor restlessness and none reported that nocturnal eating emerged after the start of dopaminergic therapy. Also, RLS patients with SRED were not significantly more likely to use dopaminergic drugs compared to RLS patients without SRED. In fact, subjects whose nocturnal eating symptoms were under control were more likely to be on these agents than subjects who continued to have nocturnal eating [70]. Fourth, a double-blind treatment trial of pramipexole for SRED demonstrated improved sleep and reduced nighttime activity and further did not result in increased feeding activity [84]. Another series monitored therapy outcomes in 44 RLS patients previously unexposed to dopaminergics. In this population, the frequency of both NE and SRED diminished by half with dopaminergics. Further, only one patient reported an exacerbation of NE after dopamine agents were initiated and there were no cases of dopaminergics inducing de novo NE. Consistent with other reports, nocturnal eating symptoms demonstrated a clinical response in parallel to motor RLS symptoms [8]. Finally, treatment with dopaminergic agents appears to improve other nonmotor manifestations of RLS that frequently coexist with SRED. In particular, all patients who reported a remission of nocturnal smoking had been treated with dopaminergic agents [84].

Conceptually, as RLS patients are predisposed to NE, greater than $60 \%$ in one survey [8], then amnestic SRED would be the expected result when RLS patients are treated with agents that suppress memory as well as executive function. Thus, it is not a surprise that $80 \%$ of RLS patients exposed to sedative-hypnotics had subsequent amnestic SRED or sleepwalking behavior [8].
While RLS is a condition distinct from PI, it can be easily misdiagnosed and thus mistreated as insomnia related to cognitive hypervigilance. In 2002, the first case series of 5 patients with zolpidem-induced amnestic SRED was reported. Incidentally, all 5 patients were noted to have RLS [16]. Others have commented that RLS appears to be ubiquitous in the setting of zolpidem induced SRED [85].

Zolpidem-induced SRED among patients with PI is rare. Among 25 PI patients treated with either a benzodiazepine or BRA, only two reported amnestic behavior, and in neither case did the events persist [8]. These findings are consistent with previous reports where SRED and SW are rare (1\% or less) in zolpidem treated insomnia patients when RLS has been carefully excluded [86]. Thus, one can conclude that in the absence of motor restlessness, sedative-hypnotics are safe agents and there is minimal risk of SW/SRED behaviors.

\section{Management of Disorders of Arousal}

\section{Evaluation}

The first steps of parasomnia management include the following: a severity assessment, identification and treatment of comorbid sleep disorders, elimination of presumed inducing agents, and ensuring environmental safety. Most CoA and many SW episodes are benign and limited in duration. In these cases, patients may be given reassurance and advised to avoid sleep deprivation and sedating agents. Situations that deserve more thorough investigation include the following: violent/ potentially injurious behavior, nonviolent dangerous behavior (such as leaving the house), high-frequency episodes, or if the parasomnia is associated with symptoms suggestive of another sleep disorder or neuropsychiatric condition [87].

Correctly diagnosing a parasomnia requires a detailed review of the sleep-wake complaints followed by a neuropsychiatric history and examination. A report from a bed partner is particularly helpful as many patients are unable to properly recall the nocturnal events by the time they are discussed with a clinician.

In SW, there is often a lifelong history, beginning in childhood, of prolonged, complex, nonviolent activities emanating from the first half of the sleep period with residual confusion [1]. However, in a series of 54 adult patients with injurious SW (and/or ST), one-third developed their persistent SW after the age of 16 years [88].

\section{Polysomnography}

The primary role in the polysomnographic evaluation of parasomnias is to rule out conditions such as sleep disordered breathing as a cause of nocturnal behaviors. The sleep fragmentation of OSA during REM or NREM sleep can lead to DEB or CoA/SW, respectively. 
PSG with video monitoring is often helpful in the evaluation of parasomnias even if abnormal behaviors do not arise during the sleep study [89]. Under routine conditions, PSG does not typically demonstrate CoA/SW or DEB. This is due to the intermittent nature of parasomnias as well as the laboratory effect (foreign environment) decreasing N3 sleep compared to the home sleeping environment $[89,90]$. However, even without abnormal behaviors, PSG facilitates diagnosis as CoA/SW patients often demonstrate NREM sleep instability.

Sleep deprivation combined with forced awakening increases the likelihood of triggering a NREM parasomnia event during laboratory testing and thus helps facilitate diagnosis. In particular, one protocol recommends $25 \mathrm{~h}$ of sleep deprivation leading up to a PSG combined with an alarm awakening during SWS. Using these methods, somnambulistic events were induced in $100 \%$ of patients with a history of SW compared to only $30 \%$ of patients if sleep deprivation is not used. Importantly, no control subject without a history of SW demonstrated somnambulistic events using this combined method of sleep deprivation and forced arousals (a 100\% sensitivity) [14].

In addition to NREM instability, CoA/SW PSG recordings often reveal a 10-s-long buildup of hypersynchronous delta waves immediately preceding a parasomnia event (see discussion on CAP above). Subsequently, postarousal EEG shows a persistence of slowed cortical activity, that either evolves into wakeful EEG activity or returns to NREM sleep [40].

Polysomnography (PSG) is used to characterize SRED with commonly consumed nocturnal food made available at bedside in order to facilitate eating behavior. Similar to SW, SRED arises out of NREM sleep. One study documented that 44 of 45 feeding episodes in 26 patients arose from NREM sleep [56].

Table 9 enlists various PSG findings in NREM parasomnia.

\section{Conservative Strategies}

\section{Environmental Safety}

Environmental modification is a critical component in treating parasomnia cases with potential for sleep-related injury. The patient should be advised to remove any bedside object or furniture that could be injurious either to them or to a bed partner. Firearms and other weapons should be removed from the bedroom and windows locked with curtains drawn to prevent lacerations. Bedroom door alarms are helpful ways to signal others that a sleepwalker is wandering, however loud auditory stimuli could paradoxically worsen NREM parasomnias [14].

\section{Treatment of Comorbid Conditions/Aggravating Factors}

Reversing comorbid conditions characterized by frequent cortical arousal in sleepwalkers often dramatically diminishes nocturnal behaviors. Sixty SW patients were studied with PSG and followed for a year. A high number $(n=53)$ were diagnosed as having sleep disordered breathing. The majority of patients had only a mild burden of disease, often not reaching criteria for OSA, but instead upper airway resistance syndrome. However, the results were striking. Only 3 patients dropped out of the study while of the remaining 50 all reported resolution of SW after treatment (42 reported CPAP, 8 upper airway surgeries). These dramatic results suggest that treatment of even mild, asymptomatic SDB may result in resolution of SW [42].

When SW is associated with sedative-hypnotics, it is of particular importance to reconsider the diagnosis for which the medication was originally prescribed. In these cases, patients may not have insomnia (for which the sedating agent was prescribed) but rather another disorder of sleep initiation such as RLS or a delayed circadian rhythm [8, 14, 33, 46, 67]. Discontinuing offending agents will typically resolve the parasomnia particularly if another underlying condition is identified and treated $[8,33,46]$. Treatments include the following: dopamine agonists in RLS [91] and evening melatonin/morning light therapy in a delayed circadian rhythm [92]. Conversely, in the setting of carefully diagnosed insomnia patients, those in whom other disorders are excluded, BRAs are well tolerated and SW rarely induced [8]. Patients with insomnia can also be successfully treated with cognitive behavioral therapy $[93,94]$. Treatment of NREM parasomnias is summarized in Table 10.
Table 9 PSG characteristics of NREM parasomnias

\begin{tabular}{lllll}
\hline Parasomnia & $\begin{array}{l}\text { Sleep } \\
\text { stage }\end{array}$ & $\begin{array}{l}\text { NREM } \\
\text { instability }\end{array}$ & $\begin{array}{l}\text { REM } \\
\text { atonia }\end{array}$ & Other features \\
\hline CoA & NREM & + & + & Hypersynchronous EEG delta activity \\
SW & NREM & + & + & RLS/PLM \\
ST & NREM & + & + & Tachycardia \\
SRED & NREM & + & + & RLS/PLM, rhythmic masticatory muscle \\
& & & & activity \\
\hline
\end{tabular}


Table 10 Treatment of NREM parasomnia

\begin{tabular}{lll}
\hline Parasomnia & Nonpharmacological [95] & Pharmacological \\
\hline CoA & $\begin{array}{l}\text { 1. Treatment of coexistent sleep conditions/ } \\
\text { aggravating factors (OSA, RLS) }\end{array}$ & Clonazepam [96] \\
& $\begin{array}{l}\text { 2. Anticipatory awakening } \\
\text { 3. Hypnosis }\end{array}$ & \\
1. Treatment of coexistent sleep conditions/ & Clonazepam [96] \\
SW & 2. Angravating factors (OSA, RLS) & \\
& $\begin{array}{l}\text { 3. Hypnosis } \\
\text { 4. Environmental safety } \\
\text { 1. Treatment of coexistent sleep conditions/ }\end{array}$ & \\
& aggravating factors & Imipramine, trazodone [97], \\
2. Counseling & paroxetine [55] \\
ST & 3. Anticipatory awakening & \\
4. Hypnosis & \\
1. Treatment of coexistent sleep conditions/ & \\
& aggravating factors (OSA, RLS) & Sertraline [98] \\
2. Hypnosis & Topiramate [99] \\
1. Treatment of coexistent sleep conditions/ & aggravating factors (OSA, RLS) & Dopamine agonists [84] \\
SRED & & Clonazepam, trimipramine, \\
& fluoxetine [7] \\
\hline
\end{tabular}

\section{Behavioral Therapy}

Some behavioral and cognitive strategies demonstrating some degree of benefit are discussed below.

\section{Hypnotherapy:}

There is some literature showing benefit of clinical hypnosis improving DOA. In 1991, a case series of 27 patients (8 with SW,15 with SW and ST, and 4 with ST) showed that hypnotherapy was helpful with lasting improvement in $74 \%$ of the patients. It was noted that 20 out of $23(87 \%)$ SW patients who underwent self-hypnosis training described significant improvement after greater than 6 months of follow-up [100]. Another later study demonstrated that 7 of $11(67 \%)$ sleepwalkers treated with physician-administered hypnosis described significant improvement which persisted after 5 years [101].

\section{Anticipatory awakening:}

It is a commonly used method in childhood NREM parasomnias [84] especially in cases where the occurrence of nocturnal events is highly predictable. This technique involves purposefully arousing the parasomniac 15 to $20 \mathrm{~min}$ prior to the anticipated onset of a typical episode for a duration of roughly 1 to 4 weeks. Sustained positive results in children have been reported; however, there is negligible data in adults $[101,102]$. This method appears to be a relatively low-risk therapy.
Other behavioral interventions include psychotherapy and relaxation therapy which have shown improvement in anecdotal cases [93, 95, 103].

\section{Pharmacotherapy}

If NREM parasomnia behaviors persist despite resolution of exacerbating disorders and removal of inducing agents, pharmacological interventions may be considered. The most commonly prescribed agents include benzodiazepines and to a lesser extent antidepressant medication. Efficacy depends upon which parasomnia is being treated. Antidepressants have some efficacy in the treatment of ST, whereas these agents may exacerbate SW.

It is important to recognize that the evidence for all therapies is currently based on a small number of studies, typically case reports and case series. Only rarely have there been controlled clinical investigations and sample size was typically small. Furthermore, as described below, much of the evidence is contradictory [104].

\section{Benzodiazepines}

Clonazepem is commonly used as first-line pharmacotherapy; however, studies show conflicting results. In 1996, a series of 170 patients with mixed sleep disorders (69 with SW/ST) were treated with benzodiazepines, primarily clonazepam ( $n=136)$, and followed for clinical response [96]. The vast majority of patients (86\%) reported good control after an 
average follow-up of 3.5 years. The authors reported that clonazepam efficacy was sustained with low risk of dosage escalation, with any significant dose increase, by paired $t$ test, after a mean 3.3 years of nightly therapy, and with low risk of side effects. A separate clinical case series reported on $6 \mathrm{SW}$ patients, who were initiated on clonazepam. SW was suppressed in 5 of 6 patients [105]. Conversely, a later report claims that clonazepam failed to demonstrate sustained efficacy in $5 \mathrm{SW}$ patients. This investigation carefully excluded even subtle sleep disordered breathing. After 1 year, all patients treated with clonazepam dropped out of the study and reported a persistence of SW [42].

\section{Antidepressant Medication}

Agents with strong serotonergic actions are occasionally effective in the treatment of some NREM parasomnia patients, most commonly ST (see serotonin hypothesis above). One report described two patients with a history of ST combined with SW, both of whom failed diazepam therapy but responded well to imipramine (a tricyclic antidepressant) [96]. Later, a 7-year-old girl with ST failed to respond to imipramine; however, she had a compelling therapeutic response to trazadone (a phenylpiperazine antidepressant) [97]. In contrast to these successful ST cases, a series of SW patients included 8 patients who were treated with various serotonergic agents and/or benzodiazepine. After 1 year of follow-up, all 8 patients described a persistence of SW [42].

Paroxetine appears to be particularly effective in the treatment of ST. In one report, 6 patients had a significant reduction if not outright elimination of ST events. The authors suggested that SSRIs may be uniquely effective for ST through serotonin effects on terror centers in the midbrain periaqueductal gray matter [55]. Conversely, there has been a report of paroxetine inducing SW [21]. Moreover, these findings are in contrast to a dramatic elimination of SW behavior in patients who are effectively treated for sleep disordered breathing [42].

\section{Sleep-Related Eating Disorder Treatment}

\section{Treatment of Comorbid Conditions}

The first goal in treating SRED is to eliminate implicated medications and correct comorbid sleep disorders, especially RLS. The majority of patients with drug-induced SRED notice improvement after inducing agents are discontinued [16, $58,67,68]$. It can often be controlled outright by treating comorbid RLS [8]. In cases of SRED associated with obstructive sleep apnea, continuous positive airway pressure may eliminate both the sleep disordered breathing and the nocturnal eating [106].

\section{Pharmacotherapy}

In cases without comorbid sleep disorders (or at least unrecognized comorbid sleep disorders) two classes of pharmacotherapies have been studied and appear to be potentially effective, antiseizure medication topiramate and dopaminergic agents. The original SRED case series noted that either bedtime levodopa or bromocriptine was effective in eliminating nocturnal eating in 8 patients [107]. Pramipexole, a dopamine agonist, was investigated in a small double-blind, placebocontrolled crossover trial. It was well tolerated and subjects noted improved sleep and reduced nighttime activity was documented with actigraphy [84]. An open-label trial of topiramate in four patients with nocturnal eating demonstrated positive results. The agent was well tolerated, reports of nocturnal eating were diminished, and weight loss (mean of $11.1 \mathrm{~kg}$ ) was noted in all four individuals over 8.5 months [108]. Finally, a study of 25 SRED patients on topiramate reported that 17 (68\%) of SRED patients were treatment responders. Adverse events were high however and over 12 months $41 \%$ of patients discontinued the medication [109]. Recently, a double-blind randomized control trial has demonstrated significant efficacy, supporting the use of topiramate in SRED. The symptoms were significantly reduced with topiramate ( 74.7 to $33.2 \%$ nights/week) compared to placebo (77.0 to $57.4 \%$ ) [99]. Use of SSRIs such as low dose sertraline at $25 \mathrm{mg}$ has also been noted to improved SRED demonstrated in two recent case reports [110].

\section{Parasomnia Overlap Disorder}

Parasomnia overlap disorder (POD) is a condition characterized by coexistence of REM sleep behavior disorder (RBD) and either a disorder of arousal, SRED, sexsomnia, or rhythmic movement disorder [1]. It is either idiopathic or sometimes can be associated with neurological conditions such as narcolepsy, multiple sclerosis, structural lesions in the brain, psychiatric disorders, substance abuse, and withdrawal states [1]. Even though it is considered a variant of of RBD according to ICSD-3 classification, association with phenoconversion into a neurodegenerative disease has not been established [111]. In fact, it has also been proposed that it represents a distinct parasomnia category owing to younger age of onset, more prominent features of DOA, and lack of proven association with synuclein disorders [112].

\section{Status Dissociatus}

Status dissociatus is a condition where disintegration of various stages of being, wakefulness, and NREM and REM sleep occurs, characterized by lack of orchestration of behavioral, 
neurophysiological, and autonomic markers defining different sleep stage boundaries. It is considered a subtype of RBD according to ICSD-3 [1] and was initially described in 1991 in 6 cases where multiple, ambiguous, and intermixed variable oscillations in sleep state-dependent variables were noted [113]. The PSG demonstrated admixtures of various markers of different sleep stages occurring simultaneously, suggesting breakdown of the boundaries between distinct sleep states. Clinically patient appeared to be asleep but would demonstrate complex motor behavior suggestive of dream enactment [114]. This phenomenon has been noted in conditions such as alcohol withdrawal state, narcolepsy, autoimmune encephalititis, Iglon-5 parasomnia, fatal familial insomnia, and alpha synuclein disorders [90, 115]. The pathophysiological mechanism is considered to be Gamma-butyric acid (GABA)-mediated thalamocortical dysfunction.

Agrypnia excitata is the extreme form of status dissociatus with compete disintegration of sleep state boundaries. Behaviorally it is manifested as continuous motor and autonomic hyperactivity with the patient clinically in a hallucinatory state with seemingly semi purposeful movements termed as "oneiric behavior" $[1,113]$.

\section{Summary}

Parasomnias represent abnormal behaviors and experiences that arise from sleep. They range from subclinical events only noticed by a wakeful bed partner to complex and also violent, potentially life-threatening motoric behaviors. DOA share the same pathophysiological mechanism, and hence, conservative management comprises of environment modification and safety assurance. NREM parasomnias are treated by identifying and reversing conditions that enhance homeostatic sleep drive and/or fragment sleep. Correcting RLS may help diminish sleepwalking behaviors in amnestic SRED. Sexsomnia is a subtype of DOA, which shares similar pathophysiological mechanism. If behavioral management is unsuccessful, pharmacotherapy with limited evidence can be utilized in potentially injurious NREM parasomnia cases.

POD and status dissociatus are lesser known variants of $\mathrm{RBD}$, which are mentioned in this article, as they intrude upon the NREM stage of sleep.

\section{References}

1. American Academy of Sleep Medicine. International classification of sleep disorders. 3rd ed. Darien, IL. Am Acad Sleep Med. 2014;3 rd editi.

2. GUILLEMINAULT C, KIRISOGLU C, DAROSA A, LOPES C, CHAN A. Sleepwalking, a disorder of NREM sleep instability.
Sleep Med. 2006;7(2):163-170. https://doi.org/10.1016/j.sleep. 2005.12.006

3. Irfan M, Schenck CH. Sleep-related orgasms in a 57-year-old woman: A case report. J Clin Sleep Med. 2018;14(1). https://doi. org $/ 10.5664 /$ jcsm. 6902

4. Dubessy A-L, Leu-Semenescu S, Attali V, Maranci J-B, Arnulf I. Sexsomnia: A Specialized Non-REM Parasomnia? Sleep. 2017;40(2). https://doi.org/10.1093/sleep/zsw043

5. Schenck CH, Arnulf I, Mahowald MW. Sleep and sex: what can go wrong? A review of the literature on sleep related disorders and abnormal sexual behaviors and experiences. Sleep. 2007;30(6): 683-702. https://doi.org/10.1093/sleep/30.6.683

6. Schenck CH. Update on Sexsomnia, Sleep Related Sexual Seizures, and Forensic Implications. NeuroQuantology. 2015;13(4). https://doi.org/10.14704/nq.2015.13.4.873

7. Muza R, Lawrence M, Drakatos P. The reality of sexsomnia. Curr Opin Pulm Med. 2016;22(6):576-582. https://doi.org/10.1097/ MCP.0000000000000321

8. Howell MJ, Schenck CH. Restless nocturnal eating: a common feature of Willis-Ekbom Syndrome (RLS). J Clin Sleep Med. 2012;8(4):413-419. https://doi.org/10.5664/jcsm.2036

9. EKBOM KA. Restless legs syndrome. Neurology. 1960;10:868873. https://doi.org/10.1212/wnl.10.9.868

10. Hublin C, Kaprio J, Partinen M, Heikkila K, Koskenvuo M. Prevalence and Genetics of Sleepwalking: A Population-based Twin Study. Neurology. 1997;48(1):177-181. https://doi.org/10. 1212/WNL.48.1.177

11. Dolder CR, Nelson MH. Hypnosedative-induced complex behaviours : incidence, mechanisms and management. CNS Drugs. 2008;22(12):1021-1036. https://doi.org/10.2165/0023210200822120-00005

12. Espa F, Dauvilliers Y, Ondze B, Billiard M, Besset A. Arousal reactions in sleepwalking and night terrors in adults: the role of respiratory events. Sleep. 2002;25(8):871-875. http://www.ncbi. nlm.nih.gov/pubmed/12489893

13. Szucs A, Horvath A, Anikó P. Progress in elucidating the pathophysiological basis of nonrapid eye movement parasomnias: not yet informing therapeutic strategies. Nat Sci Sleep. Published online March 2016:73. https://doi.org/10.2147/NSS.S71513

14. Pilon M, Montplaisir J, Zadra A. Precipitating factors of somnambulism: impact of sleep deprivation and forced arousals. Neurology. 2008;70(24):2284-2290. https://doi.org/10.1212/01. wnl.0000304082.49839.86

15. Lam S-P, Fong SY-Y, Yu MW-M, Li SX, Wing Y-K. Sleepwalking in psychiatric patients: comparison of childhood and adult onset. Aust N Z J Psychiatry. 2009;43(5):426-430. https://doi.org/10.1080/00048670902817703

16. Morgenthaler TI, Silber MH. Amnestic sleep-related eating disorder associated with zolpidem. Sleep Med. 2002;3(4):323-327. https://doi.org/10.1016/s1389-9457(02)00007-2

17. Liskow B, Pikalov A. Zaleplon overdose associated with sleepwalking and complex behavior. J Am Acad Child Adolesc Psychiatry. 2004;43(8):927-928. https://doi.org/10.1097/01.chi. 0000129219.66563.aa

18. Wallace DM, Maze T, Shafazand S. Sodium oxybate-induced sleep driving and sleep-related eating disorder. J Clin Sleep Med. 2011;7(3):310-311. https://doi.org/10.5664/JCSM.1082

19. Ferrándiz-Santos JA, Mataix-Sanjuan AL. Amitriptyline and somnambulism. Ann Pharmacother. 2000;34(10):1208. https://doi. org/10.1345/aph.10073

20. Oulis P, Kokras N, Papadimitriou GN, Masdrakis VG. Bupropion-induced sleepwalking. J Clin Psychopharmacol. 2010;30(1):83-84. https://doi.org/10.1097/JCP. $0 \mathrm{~b} 013 \mathrm{e} 3181 \mathrm{c} 9 \mathrm{c} 8 \mathrm{a} 2$ 
21. Kawashima T, Yamada S. Paroxetine-induced somnambulism. $J$ Clin Psychiatry. 2003;64(4):483. https://doi.org/10.4088/jcp. v64n0420e

22. Yeh Y-W, Chen C-H, Feng H-M, Wang S-C, Kuo S-C, Chen CK. New onset somnambulism associated with different dosage of mirtazapine: a case report. Clin Neuropharmacol. 32(4):232-233. https://doi.org/10.1097/WNF.0b013e318187bafc

23. Landry P, Warnes H, Nielsen T, Montplaisir J. Somnambulisticlike behaviour in patients attending a lithium clinic. Int Clin Psychopharmacol. 1999;14(3):173-175. http://www.ncbi.nlm. nih.gov/pubmed/10435770

24. Hafeez ZH, Kalinowski CM. Somnambulism induced by quetiapine: two case reports and a review of the literature. CNS Spectr. 2007;12(12):910-912. https://doi.org/10.1017/ s1092852900015698

25. Paquet V, Strul J, Servais L, Pelc I, Fossion P. Sleep-related eating disorder induced by olanzapine. J Clin Psychiatry. 2002;63(7): 597. https://doi.org/10.4088/jcp.v63n0710d

26. Chiu Y-H, Chen C-H, Shen WW. Somnambulism secondary to olanzapine treatment in one patient with bipolar disorder. Prog Neuropsychopharmacol Biol Psychiatry. 2008;32(2):581-582. https://doi.org/10.1016/j.pnpbp.2007.10.006

27. Hensel J, Pillmann F. Late-life somnambulism after therapy with metoprolol. Clin Neuropharmacol. 31(4):248-250. https://doi.org/ 10.1097/WNF.0b013e318157425a

28. Varkey BM, Varkey LM. Topiramate induced somnabulism and automatic behaviour. Indian J Med Sci. 2003;57(11):508-510. http://www.ncbi.nlm.nih.gov/pubmed/14646161

29. von Vigier RO, Vella S, Bianchetti MG. Agitated sleepwalking with fluoroquinolone therapy. Pediatr Infect Dis J. 1999;18(5): 484-485. https://doi.org/10.1097/00006454-199905000-00024

30. Casez O, Dananchet Y, Besson G. Migraine and somnambulism. Neurology. 2005;65(8):1334-1335. https://doi.org/10.1212/01. wnl.0000180937.20774.20

31. Kales JD, Kales A, Soldatos CR, Chamberlin K, Martin ED. Sleepwalking and night terrors related to febrile illness. Am J Psychiatry. 1979;136(9):1214-1215. https://doi.org/10.1176/ajp. 136.9.1214

32. Mouzas O, Angelopoulos N, Papaliagka M, Tsogas P. Increased frequency of self-reported parasomnias in patients suffering from vitiligo. Eur J Dermatol. 18(2):165-168. https://doi.org/10.1684/ ejd.2008.0355

33. Erickson J, Vaughn B V. Non-REM Parasomnia: The Promise of Precision Medicine. Sleep Med Clin. 2019;14(3):363-370. https:// doi.org/10.1016/j.jsmc.2019.05.002

34. Parrino L, Halasz P, Tassinari CA, Terzano MG. CAP, epilepsy and motor events during sleep: the unifying role of arousal. Sleep Med Rev. 2006;10(4):267-285. https://doi.org/10.1016/j.smrv. 2005.12.004

35. Steriade M, Llinás RR. The functional states of the thalamus and the associated neuronal interplay. Physiol Rev. 1988;68(3):649742. https://doi.org/10.1152/physrev.1988.68.3.649

36. Neckelmann D, Ursin R. Sleep stages and EEG power spectrum in relation to acoustical stimulus arousal threshold in the rat. Sleep. 1993;16(5):467-477. http://www.ncbi.nlm.nih.gov/pubmed/ 8378687

37. Ohayon MM, Guilleminault C, Priest RG. Night terrors, sleepwalking, and confusional arousals in the general population: their frequency and relationship to other sleep and mental disorders. J Clin Psychiatry. 1999;60(4):268-276; quiz 277. https://doi. org/10.4088/jcp.v60n0413

38. Mahowald MW, Schenck CH, Cramer Bornemann MA. Sleeprelated violence. Curr Neurol Neurosci Rep. 2005;5(2):153-158. https://doi.org/10.1007/s11910-005-0014-3

39. Zucconi M, Oldani A, Ferini-Strambi L, Smirne S. Arousal fluctuations in non-rapid eye movement parasomnias: the role of cyclic alternating pattern as a measure of sleep instability. $J$ Clin Neurophysiol. 1995;12(2):147-154. http://www.ncbi.nlm.nih.gov/ pubmed/7797629

40. Schenck CH, Pareja JA, Patterson AL, Mahowald MW. Analysis of polysomnographic events surrounding 252 slow-wave sleep arousals in thirty-eight adults with injurious sleepwalking and sleep terrors. J Clin Neurophysiol. 1998;15(2):159-166. https:// doi.org/10.1097/00004691-199803000-00010

41. Espa F, Ondze B, Deglise P, Billiard M, Besset A. Sleep architecture, slow wave activity, and sleep spindles in adult patients with sleepwalking and sleep terrors. Clin Neurophysiol. 2000;111(5): 929-939. https://doi.org/10.1016/s1388-2457(00)00249-2

42. Guilleminault C, Kirisoglu C, Bao G, Arias V, Chan A, Li KK. Adult chronic sleepwalking and its treatment based on polysomnography. Brain. 2005;128(Pt 5):1062-1069. https://doi. org/10.1093/brain/awh481

43. Busek P, Vankova J, Opavsky J, Salinger J, Stepanova I, Nevsimalova S. [Spectral analysis of the variations in heart rate and cardiac activation on waking up in sleepwalking]. Rev Neurol. 41(6):338-343. http://www.ncbi.nlm.nih.gov/pubmed/16163654

44. Gaudreau H, Joncas S, Zadra A, Montplaisir J. Dynamics of slowwave activity during the NREM sleep of sleepwalkers and control subjects. Sleep. 2000;23(6):755-760. http://www.ncbi.nlm.nih. gov/pubmed/11007442

45. Guilleminault C, Poyares D, Aftab FA, Palombini L, Abat F. Sleep and wakefulness in somnambulism: a spectral analysis study. J Psychosom Res. 2001;51(2):411-416. https://doi.org/10. 1016/s0022-3999(01)00187-8

46. Guilleminault C. Hypersynchronous slow delta, cyclic alternating pattern and sleepwalking. Sleep. 2006;29(1):14-15. http://www. ncbi.nlm.nih.gov/pubmed/16453974

47. Zadra A, Pilon M, Joncas S, Rompré S, Montplaisir J. Analysis of postarousal EEG activity during somnambulistic episodes. $J$ Sleep Res. 2004;13(3):279-284. https://doi.org/10.1111/j.1365-2869. 2004.00404.x

48. Guilleminault C, Lee JH, Chan A, Lopes M-C, Huang Y, da Rosa A. Non-REM-sleep instability in recurrent sleepwalking in prepubertal children. Sleep Med. 2005;6(6):515-521. https://oi.org/ 10.1016/j.sleep.2005.03.003

49. Terzaghi M, Sartori I, Tassi L, et al. Evidence of dissociated arousal states during NREM parasomnia from an intracerebral neurophysiological study. Sleep. 2009;32(3):409-412. https://doi. org/10.1093/sleep/32.3.409

50. Bassetti C, Vella S, Donati F, Wielepp P, Weder B. SPECT during sleepwalking. Lancet (London, England). 2000;356(9228):484485. https://doi.org/10.1016/S0140-6736(00)02561-7

51. Tassinari CA, Rubboli G, Gardella E, et al. Central pattern generators for a common semiology in fronto-limbic seizures and in parasomnias. A neuroethologic approach. Neurol Sci. 2005;26 Suppl 3:s225-32. https://doi.org/10.1007/s10072-005-0492-8

52. Tassinari CA, Cantalupo G, Högl B, et al. Neuroethological approach to frontolimbic epileptic seizures and parasomnias: The same central pattern generators for the same behaviours. Rev Neurol (Paris). 2009;165(10):762-768. https://doi.org/10.1016/j. neurol.2009.08.002

53. Braun AR, Balkin TJ, Wesenten NJ, et al. Regional cerebral blood flow throughout the sleep-wake cycle. An H2(15)O PET study. Brain. 1997;120 (Pt 7):1173-1197. https://doi.org/10.1093/brain/ 120.7.1173

54. Juszczak GR, Swiergiel AH. Serotonergic hypothesis of sleepwalking. Med Hypotheses. 2005;64(1):28-32. https://doi. org/10.1016/j.mehy.2004.06.013

55. Wilson SJ, Lillywhite AR, Potokar JP, Bell CJ, Nutt DJ. Adult night terrors and paroxetine. Lancet (London, England). 1997;350(9072):185. https://doi.org/10.1016/s0140-6736(05) 62351-3 
56. Vetrugno R, Manconi M, Ferini-Strambi L, Provini F, Plazzi G, Montagna P. Nocturnal eating: Sleep-related eating disorder or night eating syndrome? A videopolysomnographic study. Sleep. 2006;29(7):949-954. https://doi.org/10.1093/sleep/29.7.949

57. Jacobs BL, Martín-Cora FJ, Fornal CA. Activity of medullary serotonergic neurons in freely moving animals. Brain Res Brain Res Rev. 2002;40(1-3):45-52. https://doi.org/10.1016/s01650173(02)00187-x

58. Schenck, Carlos H. Zolpidem-induced sleep-related eating disorder (SRED) in 19 patients. Sleep. 2005;28.

59. Ho T, Jimenez A, Sanchez I, Seeger C, Joseph M. Sleep-Related Eating Disorder Associated with Zolpidem: Cases Compiled From a Literature Review. Sleep Med X. Published online July 2020: 100019. https://doi.org/10.1016/j.sleepx.2020.100019

60. Winkelman JW, Herzog DB, Fava M. The prevalence of sleeprelated eating disorder in psychiatric and non-psychiatric populations. Psychol Med. 1999;29(6):1461-1466. https://doi.org/10. $1017 / \mathrm{s} 0033291799008272$

61. Vetrugno R, Manconi M, Ferini-Strambi L, Provini F, Plazzi G, Montagna P. Nocturnal Eating: Sleep-Related Eating Disorder or Night Eating Syndrome? A Videopolysomnographic Study. Sleep. 2006;29(7):949-954. https://doi.org/10.1093/sleep/29.7. 949

62. Schenck CH, Hurwitz TD, Bundlie SR, Mahowald MW. Sleeprelated eating disorders: polysomnographic correlates of a heterogeneous syndrome distinct from daytime eating disorders. Sleep. 1991;14(5):419-431. https://doi.org/10.1093/sleep/14.5.419

63. Reading D. Paradox lost: midnight in the battleground of sleep and dreams. J Neurol Neurosurg Psychiatry. 2006;77(12):1387-1387. https://doi.org/10.1136/jnnp.2006.096131

64. Nadel C. Somnambulism, Bed-Time Medication and OverEating. Br J Psychiatry. 1981;139(1):79-79. https://doi.org/10. 1192/bjp.139.1.79a

65. Güneș S, Camkurt MA. Sleep-Related Eating Disorder Associated With Risperidone. J Clin Psychopharmacol. 2016;36(3):286-288. https://doi.org/10.1097/JCP.0000000000000502

66. Molina SM, Joshi KG. A case of zaleplon-induced amnestic sleeprelated eating disorder. J Clin Psychiatry. 2010;71(2):210-211. https://doi.org/10.4088/JCP.09105364bro

67. Chiang A, Krystal A. Report of two cases where sleep related eating behavior occurred with the extended-release formulation but not the immediate-release formulation of a sedative-hypnotic agent. J Clin Sleep Med. 2008;4(2):155-156. http://www.ncbi. nlm.nih.gov/pubmed/18468314

68. Wing YK, Lam SP, Li SX, Zhang J, Yu MWM. Sleep-related eating disorder and zolpidem: an open interventional cohort study. J Clin Psychiatry. 2010;71(5):653-656. https://doi.org/10.4088/ JCP.09105623gry

69. Hwang T-J, Ni H-C, Chen H-C, Lin Y-T, Liao S-C. Risk predictors for hypnosedative-related complex sleep behaviors: a retrospective, cross-sectional pilot study. J Clin Psychiatry. 2010;71(10):1331-1335. https://doi.org/10.4088/JCP. 09m05083bro

70. Provini F, Antelmi E, Vignatelli L, et al. Association of restless legs syndrome with nocturnal eating: a case-control study. Mov Disord. 2009;24(6):871-877. https://doi.org/10.1002/mds.22460

71. Rodriguez CL, Foldvary-Schaefer N. Clinical neurophysiology of NREM parasomnias. Handb Clin Neurol. 2019;161:397-410. https://doi.org/10.1016/B978-0-444-64142-7.00063-1

72. Ekbom KA. Restless legs syndrome. Neurology. 1960;10(9):868868. https://doi.org/10.1212/WNL.10.9.868

73. Berger K, Luedemann J, Trenkwalder C, John U, Kessler C. Sex and the risk of restless legs syndrome in the general population. Arch Intern Med. 2004;164(2):196-202. https://doi.org/10.1001/ archinte.164.2.196
74. Allen RP, Walters AS, Montplaisir J, et al. Restless legs syndrome prevalence and impact: REST general population study. Arch Intern Med. 2005;165(11):1286-1292. https://doi.org/10.1001/ archinte.165.11.1286

75. Paulus W, Dowling P, Rijsman R, Stiasny-Kolster K, Trenkwalder C. Update of the pathophysiology of the restlesslegs-syndrome. Mov Disord. 2007;22 Suppl 1:S431-9. https:// doi.org/10.1002/mds. 21824

76. Lavigne GJ, Kato T, Kolta A, Sessle BJ. Neurobiological mechanisms involved in sleep bruxism. Crit Rev Oral Biol Med. 2003;14(1):30-46. https://doi.org/10.1177/154411130301400104

77. Walters AS. Toward a better definition of the restless legs syndrome. The International Restless Legs Syndrome Study Group. Mov Disord. 1995;10(5):634-642. https://doi.org/10.1002/mds. 870100517

78. Provini F, Antelmi E, Vignatelli L, et al. Increased prevalence of nocturnal smoking in restless legs syndrome (RLS). Sleep Med. 2010;11(2):218-220. https://doi.org/10.1016/j.sleep.2009.05.016

79. Driver-Dunckley ED, Noble BN, Hentz JG, et al. Gambling and increased sexual desire with dopaminergic medications in restless legs syndrome. Clin Neuropharmacol. 30(5):249-255. https://doi. org/10.1097/wnf.0b013e31804c780e

80. Tippmann-Peikert M, Park JG, Boeve BF, Shepard JW, Silber MH. Pathologic gambling in patients with restless legs syndrome treated with dopaminergic agonists. Neurology. 2007;68(4):301303. https://doi.org/10.1212/01.wnl.0000252368.25106.b6

81. Nirenberg MJ, Waters C. Nocturnal eating in restless legs syndrome. Mov Disord. 2010;25(1):126-127. https://doi.org/10. 1002/mds.22797

82. Martin-Iverson MT, Dourish CT. Role of dopamine D-1 and D-2 receptor subtypes in mediating dopamine agonist effects on food consumption in rats. Psychopharmacology (Berl). 1988;96(3): 370-374. https://doi.org/10.1007/BF00216064

83. Schenck CH, Mahowald MW. Combined bupropion-levodopatrazodone therapy of sleep-related eating and sleep disruption in two adults with chemical dependency. Sleep. 2000;23(5):587-588. http://www.ncbi.nlm.nih.gov/pubmed/10947025

84. Provini F, Albani F, Vetrugno R, et al. A pilot double-blind placebo-controlled trial of low-dose pramipexole in sleep-related eating disorder. Eur J Neurol. 2005;12(6):432-436. https://doi.org/ 10.1111/j.1468-1331.2005.01017.x

85. Nzwalo H, Ferreira L, Peralta R, Bentes C. Sleep-related eating disorder secondary to zolpidem. BMJ Case Rep. 2013;2013. https://doi.org/10.1136/bcr-2012-008003

86. Holm KJ, Goa KL. Zolpidem: an update of its pharmacology, therapeutic efficacy and tolerability in the treatment of insomnia. Drugs. 2000;59(4):865-889. https://doi.org/10.2165/00003495200059040-00014

87. Wills L, Garcia J. Parasomnias: epidemiology and management. CNS Drugs. 2002;16(12):803-810. https://doi.org/10.2165/ 00023210-200216120-00002

88. Schenck CH, Milner DM, Hurwitz TD, Bundlie SR, Mahowald MW. A polysomnographic and clinical report on sleep-related injury in 100 adult patients. Am J Psychiatry. 1989;146(9):11661173. https://doi.org/10.1176/ajp.146.9.1166

89. Drakatos P, Marples L, Muza R, et al. Video polysomnographic findings in non-rapid eye movement parasomnia. J Sleep Res. 2019;28(2):e12772. https://doi.org/10.1111/jsr.12772

90. Irfan M, Schenck CH, Howell MJ. Non-rapid eye movement sleep and overlap parasomnias. Contin Lifelong Learn Neurol. 2017;23(4, SleepNeurology). https://doi.org/10.1212/CON. 0000000000000503

91. Zucconi M, Galbiati A, Rinaldi F, Casoni F, Ferini-Strambi L. An update on the treatment of Restless Legs Syndrome/Willis-Ekbom Disease: prospects and challenges. Expert Rev Neurother. 
2018;18(9):705-713. https://doi.org/10.1080/14737175.2018. 1510773

92. Smolensky MH, Hermida RC, Reinberg A, Sackett-Lundeen L, Portaluppi F. Circadian disruption: New clinical perspective of disease pathology and basis for chronotherapeutic intervention. Chronobiol Int. 2016;33(8):1101-1119. https://doi.org/10.1080/ 07420528.2016.1184678

93. Drakatos P, Marples L, Muza R, et al. NREM parasomnias: a treatment approach based upon a retrospective case series of 512 patients. Sleep Med. 2019;53:181-188. https://doi.org/10.1016/j. sleep.2018.03.021

94. Sivertsen B, Omvik S, Pallesen S, et al. Cognitive behavioral therapy vs zopiclone for treatment of chronic primary insomnia in older adults: a randomized controlled trial. JAMA. 2006;295(24):2851-2858. https://doi.org/10.1001/jama.295.24. 2851

95. Galbiati A, Rinaldi F, Giora E, Ferini-Strambi L, Marelli S. Behavioural and Cognitive-Behavioural Treatments of Parasomnias. Behav Neurol. 2015;2015. https://doi.org/10.1155/ 2015/786928

96. Schenck CH, Mahowald MW. Long-term, nightly benzodiazepine treatment of injurious parasomnias and other disorders of disrupted nocturnal sleep in 170 adults. Am J Med. 1996;100(3): 333-337. https://doi.org/10.1016/S0002-9343(97)89493-4

97. Balon R. Sleep terror disorder and insomnia treated with trazodone: a case report. Ann Clin Psychiatry. 1994;6(3):161-163. https://doi.org/10.3109/10401239409148998

98. Varghese R, Rey de Castro J, Liendo C, Schenck CH. Two Cases of Sleep-Related Eating Disorder Responding Promptly to LowDose Sertraline Therapy. J Clin Sleep Med. 2018;14(10):18051808. https://doi.org/10.5664/jcsm.7404

99. Winkelman JW, Wipper B, Purks J, Mei L, Schoerning L. Topiramate reduces nocturnal eating in sleep-related eating disorder. Sleep. 2020;43(9). https://doi.org/10.1093/sleep/zsaa060

100. Hurwitz TD, Mahowald MW, Schenck CH, Schluter JL, Bundlie SR. A retrospective outcome study and review of hypnosis as treatment of adults with sleepwalking and sleep terror. J Nerv Ment Dis. 1991;179(4):228-233. https://doi.org/10.1097/ 00005053-199104000-00009

101. Hauri PJ, Silber MH, Boeve BF. The treatment of parasomnias with hypnosis: a 5-year follow-up study. J Clin Sleep Med. 2007;3(4):369-373. http://www.ncbi.nlm.nih.gov/pubmed/ 17694725

102. Byars KC, Simon SL. American Academy of Pediatrics 2016 Safe Sleep Practices: Implications for Pediatric Behavioral Sleep Medicine. Behav Sleep Med. 2017;15(3):175-179. https://doi. org/10.1080/15402002.2017.1292726

103. Ntafouli M, Galbiati A, Gazea M, Bassetti CLA, Bargiotas P. Update on nonpharmacological interventions in parasomnias. Postgrad Med. 2020;132(1):72-79. https://doi.org/10.1080/ 00325481.2019 .1697119
104. Harris M, Grunstein RR. Treatments for somnambulism in adults: assessing the evidence. Sleep Med Rev. 2009;13(4):295-297. https://doi.org/10.1016/j.smrv.2008.09.003

105. Kavey NB, Whyte J, Resor SR, Gidro-Frank S. Somnambulism in adults. Neurology. 1990;40(5):749-749. https://doi.org/10.1212/ WNL.40.5.749

106. Schenck CH, Mahowald MW. Review of nocturnal sleep-related eating disorders. Int J Eat Disord. 1994;15(4):343-356. https://doi. org/10.1002/eat.2260150405

107. Schenck CH, Hurwitz TD, O'Connor KA, Mahowald MW. Additional categories of sleep-related eating disorders and the current status of treatment. Sleep. 1993;16(5):457-466. http:// www.ncbi.nlm.nih.gov/pubmed/8104356

108. Winkelman JW. Treatment of nocturnal eating syndrome and sleep-related eating disorder with topiramate. Sleep Med. 2003;4(3):243-246. https://doi.org/10.1016/s1389-9457(03) 00060-1

109. Winkelman JW. Efficacy and tolerability of open-label topiramate in the treatment of sleep-related eating disorder: a retrospective case series. J Clin Psychiatry. 2006;67(11):1729-1734. https:// doi.org/10.4088/jcp.v67n1109

110. Varghese R, Rey de Castro J, Liendo C, Schenck CH. Two Cases of Sleep-Related Eating Disorder Responding Promptly to LowDose Sertraline Therapy. J Clin Sleep Med. 2018;14(10):18051808. https://doi.org/10.5664/jcsm. 7404

111. Matos N, Iranzo A, Gaig C, Santamaria J. Videopolysomnographic documentation of non-rapid eye movement sleep parasomnia followed by rapid eye movement sleep behavior disorder: a parasomnia overlap disorder? Sleep Med. 2016;23: 46-48. https://doi.org/10.1016/j.sleep.2016.04.017

112. Dumitrascu O, Schenck CH, Applebee G, Attarian H. Parasomnia overlap disorder: a distinct pathophysiologic entity or a variant of rapid eye movement sleep behavior disorder? A case series. Sleep Med. 2013;14(11):1217-1220. https://doi.org/10.1016/j.sleep. 2013.06.012

113. Mahowald MW, Schenck CH. Status dissociatus-a perspective on states of being. Sleep. 1991;14(1):69-79. https://doi.org/10.1093/ sleep/14.1.69

114. Miró-Andreu A, López-Bernabé R, Garnés Sánchez MC, Maeztu Sardiña MC. Status dissociatus: The most extreme expression of state dissociation. Neurologia. 2019;34(3):209-211. https://doi. org/10.1016/j.nrl.2017.05.004

115. Antelmi E, Ferri R, Iranzo A, et al. From state dissociation to status dissociatus. Sleep Med Rev. 2016;28:5-17. https://doi.org/10. 1016/j.smrv.2015.07.003

Publisher's Note Springer Nature remains neutral with regard to jurisdictional claims in published maps and institutional affiliations. 